\title{
Efficacy of microsurgical tumor removal for treatment of patients with intracanalicular vestibular schwannoma presenting with disabling vestibular symptoms
}

\author{
Madjid Samii, MD, PhD, Hussam Metwali, MD, MSc, and Venelin Gerganov, MD, PhD \\ International Neuroscience Institute, Hannover, Germany
}

OBJECTIVE The aim of this study was to analyze the efficacy and risks of microsurgery via the hearing-preserving retrosigmoid approach in patients with intracanalicular vestibular schwannoma (VS) suffering from disabling vestibular symptoms, with special attention to vertigo.

METHODS This is a retrospective analysis of 19 patients with intracanalicular VS and disabling vestibular dysfunction as the main or only symptom (Group A). All of the patients reported having had disabling vertigo attacks. Subjective evaluation of the impairment of patients was performed before surgery, 3 weeks after surgery, 3 months after surgery, and 1 year after surgery, using the Dizziness Handicap Inventory (DHI). The main outcome measures were improvement in quality of life as measured using the $\mathrm{DHI}$, and general and functional outcomes, in particular facial function and hearing. Patient age, preoperative tumor size, preoperative $\mathrm{DHI}$ score, and preservation of the nontumorous vestibular nerve were tested using a multivariate regression analysis to determine factors affecting the postoperative $\mathrm{DHI}$ score. The Mann-Whitney U-test was used to compare the postoperative DHI score at 3 weeks, 3 months, and 1 year after surgery with a control group of 19 randomly selected patients with intracanalicular VSs, who presented without vestibular symptoms (Group B). The occurrence of early postoperative discrete vertigo attacks was also compared between groups.

RESULTS The preoperative DHI score was $\geq 54$ in all patients. All patients reported having had disabling rotational vertigo before surgery. The only significant factor to affect the $\mathrm{DHI}$ outcome 3 weeks and 3 months after surgery was the preoperative DHI score. The DHI outcome after 1 year was not affected by the preoperative DHI score. Compared with the control group, the DHI score at 3 weeks and 3 months after surgery was significantly worse. There was no significant difference between the groups after 1 year. Vertigo was improved in all patients and completely resolved after 1 year in 17 patients.

CONCLUSIONS Disabling vestibular dysfunction that affects quality of life should be considered an indication for surgery, even in otherwise asymptomatic patients with intracanalicular VS. Surgical removal of the tumor is safe and very effective in regard to symptom relief. All patients had excellent facial nerve function within 1 year after surgery, with a very good chance of hearing preservation.

https://thejns.org/doi/abs/10.3171/2016.4.JNS153020

KEY WORDS vestibular schwannoma; quality of life; facial nerve; outcome; hearing preservation; vertigo; vestibular nerve; dizziness

$\mathbf{P}$ ATIENTS with small vestibular schwannomas (VSs) usually present with unilateral progressive hearing loss, tinnitus, and balance problems. These patients also sometimes present with vestibular nerve dysfunction, which includes vertigo, dizziness, and unsteadiness, and occurs in up to $61 \%$ of all VSs. Vertigo as a presenting symptom occurs in $8.8 \%-28 \%$ of cases. ${ }^{17,20}$

Most patients with a chronic loss of vestibular function regain their balance due to the process of central vestibular compensation, which consists of multiple processes of perceptual, vestibuloocular, and vestibulospinal readjustment. ${ }^{28}$ However, this process of central compensation does not occur in small VSs due to continuous irritation and false input from the tumorous vestibular nerve or from the nearby compressed nerve. Sudden or rapid vestibular dysfunction or vertigo attacks may cause significant physical and social limitations and significantly impair the quality of life. ${ }^{23}$

A consensus on a universal reporting system for patients with VS was published by Kanzaki et al. in 2003. ${ }^{12}$

ABBREVIATIONS BAEP = brainstem auditory evoked potential; $\mathrm{DHI}=$ Dizziness Handicap Inventory; VS = vestibular schwannoma.

SUBMITTED December 25, 2015. ACCEPTED April 6, 2016.

INCLUDE WHEN CITING Published online June 17, 2016; DOI: 10.3171/2016.4.JNS153020. 
In this consensus, the vestibular symptoms were described from a quality of life perspective. A grading system was proposed and included 4 grades: Grade I, no dizziness or disequilibrium; Grade II, occasional or slight dizziness or disequilibrium; Grade III, moderate or persistent dizziness or disequilibrium; and Grade IV, severe persistent dizziness or disequilibrium. For a more detailed assessment of functional, emotional, and physical deficits that occur secondary to balance problems or vertigo, the Dizziness Handicap Inventory (DHI) score has been used, which describes the patient's perception of his/her vestibular symptoms. The DHI scores range from 0 (the best possible measured score) to 100 (the worst possible measured score). ${ }^{11}$

According to the experience of the senior author (M.S.), the resection of intracanalicular VS alleviates the vestibular symptoms in most cases. ${ }^{26}$ On the other hand, such patients are usually in otherwise very good general condition and have normal hearing. Therefore, the issues of surgical morbidity, hearing preservation, and operative complications are of paramount importance.

In this study, we aimed to systematically analyze the efficacy of microsurgical resection of intracanalicular VS via the hearing-preserving retrosigmoid approach in patients with disabling vestibular symptoms that significantly affect their quality of life. Furthermore, we attempted to evaluate the postoperative general and functional outcomes.

\section{Methods}

\section{Patient Population}

Nineteen consecutive patients with intracanalicular VSs suffered from preoperative disabling vestibular symptoms as the only or the main symptom (Group A), corresponding to Grade IV according to the classification by Kanzaki et al. ${ }^{12}$ These patients, who underwent surgery at the International Neuroscience Institute-Hannover between 2001 and 2013, were included in the current study.

A control group (Group B) consisted of 19 randomly selected patients with intracanalicular VS who did not complain of any vestibular symptoms at the time of presentation (Grade I according to the Kanzaki classification). The control group was randomly chosen (using Microsoft Excel's random selection tool) from the whole pool of patients who suffered from intracanalicular VS without vestibular symptoms.

The study was approved by the local ethics committee.

\section{Clinical Manifestations}

The average age in Group A ranged from 39 to 70 years (mean 47 years). This group included 10 women and 9 men. All patients in this series suffered from disabling vestibular symptoms (corresponding to Kanzaki Grade IV) at the time of presentation. The duration of the symptoms ranged from 2 to 48 months (average 15 months). All patients were initially diagnosed by independent otologists, who excluded inner ear diseases as a cause of the vestibular symptoms. The patients were subjected first to conservative treatment including vestibular rehabilitation programs, which failed to improve the symptoms. In
Group A, all patients had a positive Romberg's test, an inability to walk a straight line, and showed rotation in the Unterberger test.

All patients reported that they had disabling rotational vertigo and dizziness at the time of presentation. The quality of life was assessed using the total score of the DHI. The preoperative DHI score was $\geq 54$ in all patients (mean 66.3, median 62, range 54-94) (Table 1).

Eleven patients reported tinnitus. Facial nerve function was normal in all patients. The new Hannover classification (Table 2) was used to classify hearing level: 8 patients had preoperative hearing that was nearly normal (Class $\mathrm{H} 1$ ), 5 still had good hearing (Class H2), and 4 suffered from functional deafness before surgery (Table 3).

\section{Surgical Procedure}

All surgeries were performed with the same technique and following the same general principles, which have been described in detail previously. ${ }^{25}$ Briefly, all procedures were performed in the semisitting position under constant electrophysiological control. Once the posterior wall of the internal auditory canal was exposed, it was opened widely using a diamond drill and taking into consideration the locations of the common crus and the vestibule. The tumor was gently dissected from the nerves and vessels, and then resected. The nerve of origin of the tumor (one of the vestibular nerves) was transected during resection of the tumor. The other vestibular nerve was preserved as long as it appeared healthy and its preservation did not interfere with safe tumor resection. Gross-total resection of the tumor was achieved in all patients.

\section{Postoperative Rehabilitation}

Patients in Group A underwent a postoperative vestibular rehabilitation program, which usually lasted for 1 month. In 7 patients, the program extended to 3 months. The patients in the control group (Group B) did not need regular vestibular rehabilitation. We offered, however, to all patients after VS resection some balance training during the hospital stay in the early postoperative period.

\section{Statistical Analysis}

In Group A, multivariate regression analysis was performed to detect factors that can affect the DHI score at 3-week, 3-month, and 1-year intervals after surgery. The preoperative DHI score, tumor size, tumor location in the canal (medial, lateral, or middle), duration of symptoms, age of patient, sex, and preservation of the nontumorous vestibular nerve were tested as independent variables. A Mann-Whitney U-test was performed to compare the DHI scores at 3 weeks, 3 months, and 1 year between Groups $\mathrm{A}$ and $\mathrm{B}$. The occurrence of very early postoperative sporadic attacks of vertigo and dizziness, i.e., during the first 2 weeks after surgery, was compared between the groups.

\section{Results}

In 12 patients (63\%), the vertigo disappeared completely within 3 months after surgery. After 1 year of followup, 17 patients were free from vertigo attacks and only 2 
TABLE 1. The postoperative total DHI score versus the preoperative DHI score in Group A

\begin{tabular}{lcccc}
\hline Value & Preop & 3 Wks Postop & 3 Mos Postop & 1 Yr Postop \\
\hline Mean & 66.3 & 31.05 & 9.8 & 4.3 \\
\hline Median & 62 & 24 & 8 & 2 \\
\hline Range & $54-94$ & $16-64$ & $2-44$ & $0-32$ \\
\hline
\end{tabular}

patients $(10.5 \%)$ reported having occasional attacks of vertigo. However, the vertigo in these 2 patients was well controlled on medication (corresponding to Kanzaki Grades II and III)..$^{12}$

In Group A, 13 patients suffered from vestibular symptoms in the form of occasional vertigo and dizziness attacks in the first 2 weeks after surgery. Three weeks after surgery, the DHI score was improved in all patients (mean 31.05 , median 24, range 16-64). The patients showed further improvement after 3 months (DHI score: mean 9.8, median 8, range 2-44) and after 1 year (DHI score: mean 4.3 , median 2, range $0-32$ ). The DHI score after 1 year was $<4$ in 17 patients and reached 0 in 6 patients. However, 2 patients, who described occasional (yet improving) vertigo attacks, had DHI scores of 24 and 32 (Table 1). The patients showed improvement of the vestibular dysfunction within 3 months. After 1 year, all patients had negative Romberg's and Unterberger's tests. Seventeen patients could walk a straight line without problems.

The multivariate regression analysis showed that the preoperative DHI score significantly affected the postoperative score at 3 weeks and 3 months after surgery. Nevertheless, the preoperative DHI score did not affect the postoperative DHI score after 1 year. The other variables-age of patient, sex, duration of symptoms, tumor size, tumor location in the internal auditory canal, and preservation of the nontumorous vestibular nerve-did not affect the postoperative DHI scores.

A comparison with the control group (Group B) using a Mann-Whitney U-test showed that the DHI score was significantly worse in Group A at 3 weeks and 3 months. After 1 year, there was no statistically significant difference in the DHI score between groups. There was no statistically significant difference in the occurrences of sporadic vertigo or dizziness attacks in the first 2 weeks after surgery between groups.

\section{Functional Outcome}

Hearing outcome is presented in Table 3. In 10 of 13 patients (76\%), preoperative functional hearing was preserved. A common feature among patients who lost functional hearing was that before surgery, wave $\mathrm{V}$ of the brainstem auditory evoked potential (BAEP) recordings was significantly delayed and had low amplitude. In contrast, in the group with preserved functional hearing after surgery, the BAEPs were normal.

In all cases, the facial nerve was preserved anatomically. Shortly after surgery, 15 patients $(78 \%)$ had normal facial nerve function (Grade I according to House-Brackmann grading), 2 patients (10\%) had Grade II facial nerve function, $1(5 \%)$ had Grade III, and $1(5 \%)$ had Grade IV. At 1-year follow-up, the facial nerve function in all pa-
TABLE 2. The new Hannover classification

\begin{tabular}{cc}
\hline Class & Hearing Loss in Decibels ${ }^{*}$ \\
\hline $\mathrm{H} 1$ & $0-20$ \\
\hline $\mathrm{H} 2$ & $21-40$ \\
\hline $\mathrm{H} 3$ & $41-60$ \\
\hline $\mathrm{H} 4$ & $61-80$ \\
\hline $\mathrm{H} 5$ & $81-90$ \\
\hline
\end{tabular}

* The pure tone audiometry hearing loss is classified in steps of 20 decibels. Reproduced from Matthies C, Samii M: Management of 1000 vestibular schwannomas (acoustic neuromas): clinical presentation. Neurosurgery 40(1):1-10, 1997. Copyright Wolters Kluwer Health, Inc. Published with permission.

tients was excellent (House-Brackmann Grades I and II) (Table 4).

\section{Operative Complications}

Early after surgery, 1 patient (5\%) with well-pneumatized mastoid air cells had CSF rhinorrhea, which was successfully managed with lumbar drainage for 5 days. Two months later, he developed subcutaneous air collection, which required revision surgery to occlude the mastoid air cells. No other complications were registered in the series.

\section{Discussion}

Vestibular schwannoma is a benign tumor arising from the Schwann cells of the vestibular nerve. These lesions may cause variable degrees of hearing loss, tinnitus, dizziness, gait instability, vertigo, and, with large tumors, signs of brainstem and cerebellar compression, multiple cranial nerves dysfunction, and hydrocephalus. ${ }^{5,13,16-22,27,30,31}$ Vestibular schwannomas are generally slow-growing tumors that cause gradual loss of the vestibular nerve function over a long period of time. This loss of the vestibular functions is accompanied by gradual parallel central compensation. Thus, the larger tumors are associated with better quality of life after surgery due to the well-developed central compensation. ${ }^{2,4,8-10,15,16,29-31}$

According to some reports, however, the quality of life after surgery is worse than for the normal population or when nonoperative management is performed. ${ }^{2-4,6,8-10}$ This process of compensation is not complete with small

TABLE 3. Postoperative hearing plotted against preoperative hearing*

\begin{tabular}{lccccccc}
\hline & & \multicolumn{7}{c}{ Postop Hearing } \\
\cline { 2 - 8 } Preop Hearing & $\mathrm{H} 1$ & $\mathrm{H} 2$ & $\mathrm{H} 3$ & $\mathrm{H} 4$ & $\mathrm{H} 5$ & Deaf \\
\hline $\mathrm{H} 1$ & $\mathbf{8}$ & $\mathbf{3}$ & $\mathbf{4}$ & 1 & 0 & 0 & 0 \\
\hline $\mathrm{H} 2$ & $\mathbf{5}$ & $\mathbf{0}$ & $\mathbf{3}$ & 1 & 0 & 0 & 1 \\
\hline $\mathrm{H} 3$ & 1 & 0 & 0 & 1 & 0 & 0 & 0 \\
\hline $\mathrm{H} 4$ & 1 & 0 & 0 & 0 & 0 & 0 & 1 \\
\hline $\mathrm{H} 5$ & 0 & 0 & 0 & 0 & 0 & 0 & 0 \\
\hline Deaf & 4 & 0 & 0 & 0 & 0 & 0 & 4 \\
\hline
\end{tabular}

* The spectrum of useful hearing is in boldface type. The new Hannover classification is used. 
TABLE 4. Facial nerve outcome early and 1 year after surgery

\begin{tabular}{ccc}
\hline & \multicolumn{2}{c}{ Percentage of Patients } \\
\cline { 2 - 3 } H-B Facial Nerve Grade & Early Postop $(\mathrm{N}=19)^{*}$ & After $1 \mathrm{Yr}(\mathrm{N}=19)^{*}$ \\
\hline I & $78(\mathrm{n}=15)$ & $89(\mathrm{n}=17)$ \\
\hline II & $10(\mathrm{n}=2)$ & $10(\mathrm{n}=2)$ \\
\hline III & $5(\mathrm{n}=1)$ & $0(\mathrm{n}=0)$ \\
\hline IV & $5(\mathrm{n}=1)$ & $0(\mathrm{n}=0)$ \\
\hline V & $0(n=0)$ & $0(n=0)$ \\
\hline VI & $0(n=0)$ & $0(n=0)$ \\
\hline
\end{tabular}

$\mathrm{H}-\mathrm{B}=$ House-Brackmann.

* Totals do not add to $100 \%$ due to rounding.

tumors or in cases of sudden loss of the vestibular functions..$^{1,2,6,9,27-30}$ Our hypothesis is that continuous firing from irritated vestibular nerves in cases of small VSs hinders the process of central compensation and causes the intractable vestibular symptoms.

Our previous experience showed that the disabling vestibular dysfunction and vertigo in particular could be positively influenced by surgery, and that this option should be discussed and offered to patients. ${ }^{26}$ On the other hand, such patients are usually in otherwise very good general condition and have normal hearing. The issues of surgical morbidity and operative complications are hence of paramount importance. It is unacceptable to induce new functional deficits in such patients. Therefore, the hearing-destructive translabyrinthine approach for managing intracanalicular VS that causes disabling vertigo, as recommended by Godefroy et al., might be a plausible alternative only in patients with hearing loss.?

Vertigo attacks seem to be more disruptive to quality of life than permanent unsteadiness and/or dizziness. In some patients, these symptoms are unresponsive to medical treatment and cause significant physical and social limitations. However, vertigo as an indication for surgery of intracanalicular VS has been only occasionally mentioned because it is rarely the main or the only presenting symptom. ${ }^{7}$ Wagner et al. studied the outcome of the vestibular functions in relation to the tumor size. In patients with a small VS $(<2 \mathrm{~cm})$, vertigo was present in $48 \%$ of patients before surgery and in $10 \%$ of patients after surgery. ${ }^{30}$

Breivik et al. reported gradual decline of vertigo in patients with a small VS who were conservatively managed. They also reported that Gamma Knife surgery had neither a beneficial nor a negative effect on vertigo. ${ }^{3}$ Myrseth et al. did not find any improvement or worsening of the balance problem in both groups of patients with VS treated with radiosurgery or with surgery. ${ }^{21}$ Contrary to that, Régis et al. did find a difference between microsurgery and radiosurgery regarding the postoperative vertigo. Nevertheless, they did not delineate the effect of surgery or radiosurgery on this symptom. ${ }^{24}$

Intracanalicular VSs cause vertigo by affecting one of the vestibular nerves of origin. Most likely, the high intracanalicular pressure associated with tumor growth, which is correlated with effects on the cochlear nerve, ${ }^{14}$ can also aggravate the compression on the other vestibular nerve. Surgery aims for complete tumor removal and functional preservation. Because the tumor originates from one of the vestibular nerves, the nerve of tumor origin is resected with the tumor. This provides a deafferentation that is similar to the mechanism of vestibular neurectomy in the management of intractable vertigo of Meniere's disease.

The other nerve is preserved as long as it is healthy. In some cases, the nontumorous vestibular nerve is flattened on the tumor surface. In these cases, the nerve is transected to allow safe tumor removal and to avoid excessive manipulation that could endanger the cochlear and facial nerves. The resection of the tumor mass, as well as the opening of the canal, alleviates the pressure on the healthy vestibular nerve. Thus, we could hypothesize that the positive effect of surgery on vertigo is due to both the deafferentation of the unhealthy vestibular nerve and the decompression of the healthy one. Accordingly, the unstable vestibular impulses to the vestibular nuclei will be stabilized.

Godefroy et al. proposed complete vestibular deafferentation by labyrinthectomy and transection of both vestibular nerves to stabilize incoming signals to the vestibular nuclei. According to our results, and in contrast to the concept of Godefroy et al., complete deafferentation is not required if the nontumorous vestibular nerve is healthy. None of the 7 patients with preserved nontumorous vestibular nerves experienced persistent postoperative vertigo for longer than 3 months or worsening of their preoperative vertigo.

We designed this study to evaluate the efficacy of the hearing-preserving retrosigmoid surgery in regard to disabling vestibular dysfunction in patients with intracanalicular VSs, who presented with disabling vertigo as their only or main symptom. Thirteen patients complained about sporadic attacks of dizziness and vertigo in the first 2 weeks after surgery. The incidence of the sporadic postoperative attacks of vertigo and dizziness was not significantly different between Groups A and B. It could be because sudden changes in vestibular input after surgery can induce vertigo and dizziness.

Vestibular symptoms and patient perception of the vestibular dysfunction in terms of quality of life were continuously improving at 3 weeks, 3 months, and 1 year after surgery. Only 2 patients (from Group A) had intermittent, yet improving, symptoms of vestibular dysfunction after 1 year. The DHI score of Group A was significantly worse than for Group B after 3 weeks and 3 months. After 1 year, there was no statistical difference between the groups. This can be explained by completion of the process of central compensation after stabilization of the vestibular input, as well as by the role of vestibular rehabilitation.

Vestibular rehabilitation, although it failed before tumor resection, can enhance the central vestibular compensation after tumor resection and stabilization of the vestibular impulse. All patients in Group A were enrolled in vestibular rehabilitation programs. The patients in Group $\mathrm{B}$ were not treated with prolonged vestibular rehabilitation programs. Balance training is performed routinely in the early postoperative phase in all patients who undergo surgery for VS in our institution.

The multivariate regression analysis showed that the preoperative DHI score significantly affected the postoperative score at 3 weeks and 3 months after surgery. Nevertheless, the preoperative DHI score did not affect the DHI score after 1 year. The other variables (age of patient, 
sex, duration of symptoms, tumor size, and preservation of the nontumorous vestibular nerve) did not affect the postoperative DHI score. The more severe the preoperative vestibular symptoms as measured by the DHI score, the worse the DHI score was after 3 weeks and 3 months. The DHI score after 1 year was not influenced by the preoperative DHI score, as explained by the fact that almost all patients were free of symptoms after 1 year.

Because of the tumor size in this group, radiosurgery could be an attractive alternative. To the best of our knowledge, there is no systematic analysis of a similar group of patients, who presented with disabling vestibular symptoms, when treated with radiosurgery. Clear evidence is missing regarding the alleviation or worsening of disabling vestibular symptoms, as well as the long-term outcome if this group of patients were to be treated with radiosurgery. Further investigations are warranted.

In regard to the outcome, our study showed that surgery is safe and does not expose the patient to the risk of major lasting functional deficits. This finding is very important considering the fact that all patients at presentation had normal facial nerve function, and 68\% presented with functional hearing. In this group, functional hearing was preserved in 10 of 13 patients (77\%). We could identify which patients were at risk for hearing loss by using a low-amplitude delayed V wave of the BAEP, which is important for their preoperative counseling. The facial nerve outcome was excellent in all patients. Within 1 year, it was completely normal (House-Brackmann Grade I) in 17 patients, and 2 patients had minimal dysfunction (HouseBrackmann Grade II). Still, 4 patients had some degree of facial dysfunction early after surgery. Although function was recovered later, this possibility has to be explained to the patient and his/her family.

\section{Study Limitations}

The main limitation of this study is the small sample size. The number of patients with VS who suffer from intractable disabling vestibular symptoms is relatively low. The patients in this study were initially diagnosed by independent otologists, who did the detailed vestibular laboratory investigations. Unfortunately, the details of these investigations were not available to us in the majority of cases. Due to the lack of these details, documentation of the central compensation via these tests was not possible. We did, however, use clinical evidence in the form of subjective perception of the improvement (i.e., DHI score) and clinical evaluation. We did not perform postoperative laboratory tests of the vestibular function. The main assessment was directed toward the clinical outcome.

\section{Conclusions}

Disabling vestibular symptoms should be considered an indication for surgery, even in otherwise asymptomatic patients with intracanalicular VS. Surgical removal of the tumor is very effective in regard to relief of the vestibular symptoms. Patients who presented with a high DHI score had worse DHI scores at 3 weeks and 3 months. After 1 year, the DHI score was significantly improved. Surgery for treatment of such tumors is safe, and the risk of surgery-related complications is very low. All patients regained excellent facial nerve function within 1 year. In cases where preoperative hearing is at a functional level and the BAEP trace is normal, functional hearing can be preserved.

\section{References}

1. Bademci G, Batay F, Yorulmaz I, Küçük B, Cağlar S: Selective microsurgical vestibular neurectomy: an option in the treatment of intractable vertigo and related microsurgical landmarks. Minim Invasive Neurosurg 47:54-57, 2004

2. Batuecas-Caletrio A, Santacruz-Ruiz S, Muñoz-Herrera A, Sousa P, Otero A, Perez-Fernandez N: Vestibular compensation after vestibular schwannoma surgery: normalization of the subjective visual vertical and disability. Acta Otolaryngol 133:475-480, 2013

3. Breivik CN, Nilsen RM, Myrseth E, Pedersen PH, Varughese JK, Chaudhry AA, et al: Conservative management or gamma knife radiosurgery for vestibular schwannoma: tumor growth, symptoms, and quality of life. Neurosurgery 73:48-57, 2013

4. da Cruz MJ, Moffat DA, Hardy DG: Postoperative quality of life in vestibular schwannoma patients measured by the SF36 Health Questionnaire. Laryngoscope 110:151-155, 2000

5. Driscoll CL, Green JD, Beatty CW, McCaffrey TV, Marrs CD: In vitro characteristics of a glass ionomer cement. Skull Base Surg 8:175-180, 1998

6. El-Kashlan HK, Shepard NT, Arts HA, Telian SA: Disability from vestibular symptoms after acoustic neuroma resection. Am J Otol 19:104-111, 1998

7. Godefroy WP, Hastan D, van der Mey AG: Translabyrinthine surgery for disabling vertigo in vestibular schwannoma patients. Clin Otolaryngol 32:167-172, 2007

8. Herdman SJ, Clendaniel RA, Mattox DE, Holliday MJ, Niparko JK: Vestibular adaptation exercises and recovery: acute stage after acoustic neuroma resection. Otolaryngol Head Neck Surg 113:77-87, 1995

9. Humphriss RL, Baguley DM, Moffat DA: Change in dizziness handicap after vestibular schwannoma excision. Otol Neurotol 24:661-665, 2003

10. Inoue Y, Ogawa K, Kanzaki J: Quality of life of vestibular schwannoma patients after surgery. Acta Otolaryngol 121:59-61, 2001

11. Jacobson GP, Newman CW: The development of the Dizziness Handicap Inventory. Arch Otolaryngol Head Neck Surg 116:424-427, 1990

12. Kanzaki J, Tos M, Sanna M, Moffat DA, Monsell EM, Berliner KI: New and modified reporting systems from the consensus meeting on systems for reporting results in vestibular schwannoma. Otol Neurotol 24:642-649, 2003

13. Kentala E, Pyykkö I: Clinical picture of vestibular schwannoma. Auris Nasus Larynx 28:15-22, 2001

14. Lapsiwala SB, Pyle GM, Kaemmerle AW, Sasse FJ, Badie B: Correlation between auditory function and internal auditory canal pressure in patients with vestibular schwannomas. J Neurosurg 96:872-876, 2002

15. Li CW, Cousins V, Hooper R: Vestibulo-ocular compensation following unilateral vestibular deafferentation. Ann Otol Rhinol Laryngol 101:525-529, 1992

16. Martin HC, Sethi J, Lang D, Neil-Dwyer G, Lutman ME, Yardley L: Patient-assessed outcomes after excision of acoustic neuroma: postoperative symptoms and quality of life. $\mathbf{J}$ Neurosurg 94:211-216, 2001

17. Matthies C, Samii M, Krebs S: Management of vestibular schwannomas (acoustic neuromas): radiological features in 202 cases - their value for diagnosis and their predictive importance. Neurosurgery 40:469-482, 1997 
18. Moffat DA, Baguley DM, Beynon GJ, Da Cruz M: Clinical acumen and vestibular schwannoma. Am J Otol 19:82-87, 1998

19. Møller P, Myrseth E, Pedersen PH, Larsen JL, Krakenes J, Moen G: Acoustic neuroma-treatment modalities. Surgery, gamma-knife or observation? Acta Otolaryngol Suppl 543:34-37, 2000

20. Morrison GA, Sterkers JM: Unusual presentations of acoustic tumours. Clin Otolaryngol Allied Sci 21:80-83, 1996

21. Myrseth E, Møller P, Pedersen PH, Lund-Johansen M: Vestibular schwannoma: surgery or Gamma Knife radiosurgery? A prospective, nonrandomized study. Neurosurgery 64:654-663, 2009

22. Myrseth E, Møller P, Wentzel-Larsen T, Goplen F, LundJohansen M: Untreated vestibular schwannomas: vertigo is a powerful predictor for health-related quality of life. Neurosurgery 59:67-76, 2006

23. Parietti-Winkler C, Gauchard GC, Simon C, Perrin PP: Sensorimotor postural rearrangement after unilateral vestibular deafferentation in patients with acoustic neuroma. Neurosci Res 55:171-181, 2006

24. Régis J, Pellet W, Delsanti C, Dufour H, Roche PH, Thomas$\sin \mathrm{JM}$, et al: Functional outcome after gamma knife surgery or microsurgery for vestibular schwannomas. J Neurosurg 97:1091-1100, 2002

25. Samii M, Matthies C: Management of 1000 vestibular schwannomas (acoustic neuromas): surgical management and results with an emphasis on complications and how to avoid them. Neurosurgery 40:11-23, 1997

26. Samii M, Matthies C, Tatagiba M: Intracanalicular acoustic neurinomas. Neurosurgery 29:189-199, 1991

27. Sughrue ME, Yang I, Aranda D, Lobo K, Pitts LH, Cheung SW, et al: The natural history of untreated sporadic vestibular schwannomas: a comprehensive review of hearing outcomes. J Neurosurg 112:163-167, 2010
28. Taylor J, Goodkin HP: Dizziness and vertigo in the adolescent. Otolaryngol Clin North Am 44:309-321, vii-viii, 2011

29. Uehara N, Tanimoto H, Nishikawa T, Doi K, Katsunuma S, Kimura $\mathrm{H}$, et al: Vestibular dysfunction and compensation after removal of acoustic neuroma. J Vestib Res 21:289-295, 2011

30. Wagner JN, Glaser M, Wowra B, Muacevic A, Goldbrunner $\mathrm{R}$, Cnyrim C, et al: Vestibular function and quality of life in vestibular schwannoma: does size matter? Front Neurol 2:55, 2011

31. Walsh RM, Bath AP, Bance ML, Keller A, Tator CH, Rutka JA: The natural history of untreated vestibular schwannomas. Is there a role for conservative management? Rev Laryngol Otol Rhinol (Bord) 121:21-26, 2000

\section{Disclosures}

The authors report no conflict of interest concerning the materials or methods used in this study or the findings specified in this paper.

\section{Author Contributions}

Conception and design: Metwali. Acquisition of data: Metwali. Analysis and interpretation of data: Metwali. Drafting the article: Metwali. Critically revising the article: all authors. Reviewed submitted version of manuscript: all authors. Approved the final version of the manuscript on behalf of all authors: Metwali. Statistical analysis: Metwali. Study supervision: Samii, Gerganov.

\section{Correspondence}

Hussam Metwali, International Neuroscience Institute, Rudolf Pichlmayr Str. 4, 30625 Hannover, Germany. email: drhussamm@yahoo.com. 